\title{
Diversidad genética de cepas de Actinobacillus pleuropneumoniae (App) aisladas desde planteles de producción intensiva de cerdos en Chile
}

\author{
Genetic diversity of Actinobacillus pleuropneumoniae (App) strains in intensive \\ swine farms in Chile \\ V Neira-Ramírez ${ }^{\text {* }}$, M Quezada ${ }^{\mathrm{b}}, \mathbf{R}$ Cubillos $^{\mathbf{b}}, \mathbf{A}$ Ruiz $^{\mathrm{b}}$ \\ aPrograma de Doctorado en Ciencias Veterinarias, Facultad de Ciencias Veterinarias, \\ Universidad de Concepción, Chillán, Chile. \\ ${ }^{\text {b}}$ Departamento de Patología y Medicina Preventiva, Facultad de Ciencias Veterinarias, \\ Universidad de Concepción, Chillán, Chile.
}

\begin{abstract}
SUMMARY
Actinobacillus pleuropneumoniae (App) is the etiologic agent of porcine contagious pleuropneumonia, an important bacterial disease in intensive pig production. In the world were described 15 App serotypes, in Chile serotypes 1 and 5 have been reported. The serotyping technique is slow, expensive and difficult; currently, a molecular tool named PCR is available to "serotyping" by Apx toxins genotyping, which is quick, non-expensive and easy. 60 App strains were evaluated, isolated from 9 intensive swine farms distributed in Chile. These isolates were obtained from lungs of pigs with lesions of porcine contagious pleuropneumonia. All the strains were isolated during the years 2007, 2008 and 2009, by means of conventional bacteriology and confirmed by API. A multiplex PCR for Apx toxin genes was performed to differentiate the App serotypes. The results showed genotypes detected only for serotypes 4, 6 and 7, first described in Chile. The most frequently isolated was serotype 7. Also, there was no predominant serotype in the different geographical zones, except in the regions of O'Higgins and Biobío where it was most frequently isolated serotype 7. The present study is the first approach in order to know the distribution of App serotypes in Chile. Studies including greater numbers of farms and isolates per farm are necessary to know the real diversity of App serotypes and genotypes in Chilean swine production farms.
\end{abstract}

Palabras clave: Actinobacillus pleuropneumoniae, serotipificación, genotipificación.

Key words: Actinobacillus pleuropneumoniae, serotyping, genotyping.

\section{INTRODUCCIÓN}

Actinobacillus pleuropneumoniae (App) es el agente etiológico de la pleuroneumonía contagiosa porcina (PCP) (Savoye y col 2000), una de las enfermedades bacterianas más importantes en producción porcina intensiva y parte del complejo respiratorio porcino (Thacker y Thanawongnuwech 2002). La PCP provoca elevadas pérdidas económicas, debido a la alta mortalidad causada en cuadros agudos y debido a la baja de los parámetros productivos en cuadros crónicos y subclínicos (Gottschalk y Taylor 2006).

La PCP es una enfermedad ubicua. Se han descrito brotes en todos los países, aunque actualmente constituye un problema crítico para la gran mayoría de los países sudamericanos; en cambio en Europa y Norteamérica la enfermedad se encuentra controlada (Gottschalk y Taylor 2006). A la fecha, se han descrito 15 serotipos de acuerdo al tipo de antígeno capsular (Nielsen y col 1997, Haesebrouck y col 1997). Algunos de estos serotipos generan reacciones cruzadas cuando son serotipificados

Aceptado: 05.04.2012.

* vneira@udec.cl de la forma tradicional, dificultando la interpretación de resultados, incluyendo que la técnica es tediosa y cara. Se ha observado que la mayoría de los distintos serotipos capsulares de App presentan un perfil genético de toxinas particular. Esto permite inferir el serotipo de la mayoría de los aislados mediante técnicas moleculares rápidas como la reacción en cadena de la polimerasa de genes que codifican para las toxinas Apx, tal como la descrita por Rayamajhi y col en 2005. Este método permite desarrollar una técnica homologable a la serotipificación de manera rápida, económica y al alcance de cualquier laboratorio de diagnóstico, permitiendo así realizar estudios de caracterización en gran cantidad de aislados generando información valiosa de la epidemiología de la bacteria (Rayamajhi y col 2005).

En Chile hay escasa información acerca de los serotipos prevalentes de App, sólo se han publicado dos trabajos, ambos sobre brotes de PCP en planteles porcinos de la Región Metropolitana, en los que se determinó la participación de los serotipos 1 y 5 (Sánchez y col 1986, Olivares y Morgado 1988). El objetivo del presente estudio fue determinar los serotipos de App mediante una nueva técnica basada en PCR, en casos confirmados de PCP obtenidos desde sistemas de producción intensiva de cerdos en Chile. 


\section{MATERIAL Y MÉTODOS}

\section{UNIDAD MUESTRAL}

Para el estudio se utilizaron 60 aislados de campo de App, recopilados entre los años 2007 y 2009, provenientes desde sistemas de producción intensiva pertenecientes a nueve empresas productoras de cerdos del país, con infección endémica de App, las que representan al 23,5\% del total de la industria nacional y cuya población de animales representó en el año 2010 a más del $94 \%$ de la producción de cerdos de Chile. Los planteles representados están ubicados desde la Región Metropolitana hasta la Región del Biobío, donde se encuentra la mayoría de los sistemas de producción intensiva de cerdos en Chile.

Para cada aislado se registró el año de aislamiento y zona geográfica de procedencia (cuadro 1). En detalle, el año 2007 se obtuvieron aislados desde cinco empresas ubicadas en las regiones de O’Higgins, Maule y Biobío, recopilando un total de 15 aislados (25\% total del estudio). Durante el año 2008 se aisló App desde siete empresas porcinas de las regiones de O’Higgins, Maule, Biobío y Metropolitana, recopilándose en total 24 aislados (40\% total del estudio). Finalmente, para el año 2009 se recopilaron 21 aislados de App (35\% total del estudio) provenientes de seis empresas porcinas, ubicadas en las regiones de O'Higgins, Maule, Biobío y Metropolitana, respectivamente.

Todos los aislados fueron obtenidos de cerdos afectados con cuadro clínico PCP en fase de engorda, diagnosticados siguiendo el procedimiento bacteriológico estándar y confirmadas mediante la prueba API 20 NE (Bio-Meriuex, Marcy l'Étoile, France) en el Laboratorio de Diagnóstico Porcino, perteneciente al Departamento de Patología y Medicina Preventiva de la Facultad de Ciencias Veterinarias de la Universidad de Concepción, Campus Chillán, Chile.

\section{TÉCNICA DE PCR}

Se extrajo el material genético a partir de cultivo puro de App utilizando el kit comercial Prepman Ultra ${ }^{\circledR}$, siguiendo el protocolo del fabricante. Luego se realizaron dos reacciones de PCR, una de diagnóstico de rutina confirmatoria del patógeno, realizada siguiendo el protocolo de Schaller y col (2001) y la segunda para realizar la caracterización genética de los aislados, mediante un PCR multiplex, amplificando los genes que codifican las toxinas ApxI, ApxII, ApxIII y ApxIV según el protocolo descrito por Rayamajhi y col 2005 (cuadro 2). En detalle, este protocolo utiliza las diferencias genéticas de los diferentes aislados para agruparlos en 11 perfiles genéticos que se relacionan con los 15 serotipos descritos a la fecha. Así, permite diferenciar claramente los serotipos 1, 3, $4,5,6,7,10,14$ y presenta tres perfiles inespecíficos que pueden representar a más de un serotipo, existiendo inespecificidad entre los serotipos 2,8 y 15; 9 y 11; 12 y 13 (cuadro 2).
Cuadro 1. Identificación de los aislados utilizados en el estudio. Identification of isolates evaluated.

\begin{tabular}{lclc}
\hline Año & Plantel & \multicolumn{1}{c}{ Ubicación } & N $^{\text {o }}$ \\
\hline 2007 & A & Región O’Higgins & 3 \\
2007 & B & Región O'Higgins & 3 \\
2007 & C & Región del Maule & 1 \\
2007 & D & Región del Biobío & 7 \\
2007 & E & Región del Biobío & 1 \\
2008 & A & Región O’Higgins & 4 \\
2008 & C & Región del Maule & 2 \\
2008 & D & Región del Biobío & 3 \\
2008 & E & Región del Biobío & 6 \\
2008 & F & Región del Maule & 3 \\
2008 & G & R. Metropolitana & 1 \\
2008 & I & R. Metropolitana & 5 \\
2009 & A & Región O’Higgins & 2 \\
2009 & B & Región O’Higgins & 2 \\
2009 & C & Región del Maule & 2 \\
2009 & D & Región del Biobío & 11 \\
2009 & E & Región del Biobío & 3 \\
2009 & H & R. Metropolitana & 1 \\
\hline
\end{tabular}

La técnica de PCR multiplex se desarrolló mediante una mezcla maestra que consta de $2,5 \mu \mathrm{l}$ de buffer $10 \mathrm{x}, 1 \mu \mathrm{l}$ de DNA templado, 1,5 mM de $\mathrm{MgCl}_{2}, 0,2 \mathrm{mM}$ de cada trifosfato desoxinucleósido (dNTP), 0,2 $\mu \mathrm{M}$ de cada primer y 1,25 U de Taq polimerasa en un volumen final de $25 \mu \mathrm{l}$. El programa de termociclado consta de una desnaturalización inicial a $94^{\circ} \mathrm{C}$ por 5 minutos, posteriormente 35 ciclos que constan de una fase de desnaturalización a $94{ }^{\circ} \mathrm{C}$ por 30 segundos, una fase de anillado a $55^{\circ} \mathrm{C}$ por 30 segundos y una fase de elongación a $72{ }^{\circ} \mathrm{C}$ por 180 segundos. Finalmente, la muestra es sometida a una fase de elongación a $72{ }^{\circ} \mathrm{C}$ por 10 minutos, para luego ser conservada a $4{ }^{\circ} \mathrm{C}$. Este programa fue realizado en un termociclador Eppendorf $₫$ JM Research, Modelo TTC-200. Finalmente, los productos fueron sometidos a una electroforesis en un gel de agarosa al $2 \%$, teñido con bromuro de etidio. Las bandas de DNA amplificado se observaron en un transiluminador de rayos UV (M-15, UVP). En cada reacción de PCR multiplex realizada se incluyeron controles positivos correspondientes a ADN de cepas de referencia de los serotipos 1, 2, 4 y 6 , que permiten en conjunto amplificar todos los productos posibles de la técnica (cuadro 2).

\section{ANÁLISIS ESTADÍSTICO}

En el estudio se realizó estadística descriptiva mediante frecuencias absolutas y relativas. Se utilizó el test exacto de Fisher para analizar las frecuencias de distribución de los serotipos por empresa, año de aislamiento y zona geográfica. Adicionalmente, se usó el mismo test para 
Cuadro 2. Nombre de los diferentes genes Apx y su correspondiente número de pares de bases, utilizados para genotipificar y "serotipificar" las cepas de App.

Names of different Apx genes and the expected sizes used for the App genotyping and "serotyping".

\begin{tabular}{|c|c|c|c|c|c|c|c|c|}
\hline Serotipo & $\begin{array}{l}\text { ApxIA } \\
\text { (723bp) }\end{array}$ & $\begin{array}{l}\text { ApxIB } \\
\text { (811bp) }\end{array}$ & $\begin{array}{l}\text { ApxII } \\
\text { (965bp) }\end{array}$ & $\begin{array}{l}\text { ApxIII } \\
\text { (396bp) }\end{array}$ & $\begin{array}{l}\text { ApxIV } \\
\text { (1600bp) }\end{array}$ & $\begin{array}{c}\text { ApxIV } \\
\text { (2000bp) }\end{array}$ & $\begin{array}{c}\text { ApxIV } \\
\text { (2400bp) }\end{array}$ & $\begin{array}{c}\text { ApxIV } \\
\text { (2800bp) }\end{array}$ \\
\hline 1 & + & + & + & & & & + & \\
\hline 2 & & + & + & + & & & & + \\
\hline 3 & & & + & + & & & + & \\
\hline 4 & & + & + & + & + & & & \\
\hline 5 & + & + & + & & & & & + \\
\hline 6 & & + & + & + & & + & & \\
\hline 7 & & + & + & & & & & + \\
\hline 8 & & + & + & + & & & & + \\
\hline 9 & + & + & + & & + & & & \\
\hline 10 & + & + & & & & & & + \\
\hline 11 & + & + & + & & + & & & \\
\hline 12 & & + & + & & & & + & \\
\hline 13 & & + & + & & & & + & \\
\hline 14 & + & + & & & & & + & \\
\hline 15 & & + & + & + & & & & + \\
\hline
\end{tabular}

(+): Serotipo positivo a la amplificación genética.

determinar la existencia de predominancia de los serotipos identificados dentro y entre empresas, así como también en las zonas geográficas de procedencia.

\section{RESULTADOS}

Todos los aislados evaluados fueron diagnosticados como A. pleuropneumoniae mediante el PCR confirmatorio, concordando con lo encontrado mediante las pruebas bioquímicas desarrolladas por el Laboratorio de Diagnóstico Porcino. Asimismo, fue posible realizar la caracterización molecular, mediante el PCR multiplex, en la totalidad de las cepas estudiadas.

Los 60 aislados evaluados fueron agrupados en 3 de los 11 perfiles posibles de determinar mediante la técnica utilizada, observándose los genotipos correspondientes a los serotipos 4, 6 y 7 (figura 1). En detalle, el perfil genético correspondiente al serotipo 7 fue el más frecuentemente encontrado, observándose en el $60 \%$ de los aislados $(\mathrm{P}<0,05)$, un $28 \%$ correspondió al perfil del serotipo 6 y solo un $12 \%$ fue caracterizado como serotipo 4 (cuadro 3).

En todos los años de estudio fueron descritos los serotipos 4, 6 y 7. En detalle el perfil genético correspondiente al serotipo 7 fue el más frecuentemente encontrado en todos los años, en 2007 (53\%), 2008 (50\%) y 2009 (76\%); sin embargo, estadísticamente se demostró que sólo en el año 2009 fue significativamente mayor que los serotipos 4 y 6 (cuadro 3 ).

Por otro lado, los tres serotipos descritos fueron encontrados en todas las regiones de estudio. La presentación de ellos en la Región Metropolitana y Región de Maule no evidenció ningún serotipo predominante, al contrario, en las regiones de O'Higgins y del Biobío, el serotipo 7 obtuvo una mayor frecuencia de aislamiento, siendo esta estadísticamente significativa $(\mathrm{P}<0,05)$.

Los resultados por empresa mostraron que en 4 de las 9 empresas (A, C, D y F) evidenciaron los perfiles correspondientes a los serotipos 4, 6 y 7 (cuadro 3). Por otro lado, otras tres empresas (B, E e I) presentaron el perfil correspondiente al serotipo 7 más el del serotipo 4

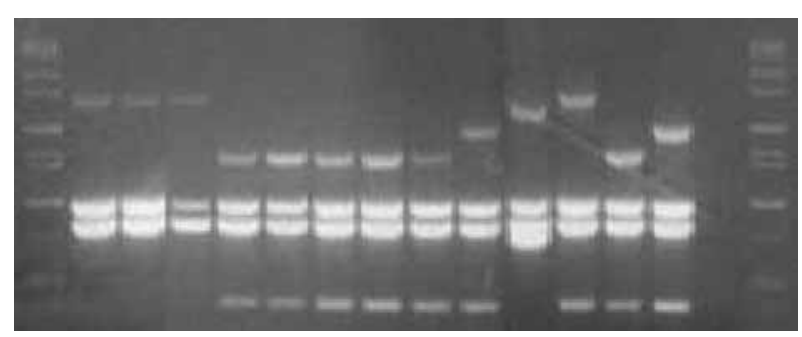

Figura 1. Resultado de serotipificación con perfiles genéticos a través, PCR multiplex de genes Apx de App. Carril 1, estándar de pares de bases; Carriles 2 al 4, aislados correspondientes al patrón de amplificación de serotipo 7; Carriles 5 al 9, aislados correspondientes al patrón de amplificación de serotipo 4; Carril 10, aislado correspondiente al patrón de amplificación de serotipo 6; Carriles 11, 12, 13 y 14, controles positivos de serotipos 1, 2, 4 y 6 respectivamente; Carril 15, control negativo; Carril 16, estándar de pares de bases.

Serotyping App results mean multiplex PCR of App Apx genes. Lane 1, ladder; lanes 2 to 4 , serotype 7 isolates; lanes 5 to 9 , corresponding to serotype 4 isolates; lane 10, serotype 6 isolate; lanes 11 , 12,13 and 14, positive controls for serotypes 1, 2, 4 and 6 respectively; lane 15 , negative control; lane 16 , ladder. 
Cuadro 3. Resumen de resultados de acuerdo a empresa, año y región.

Summary of results according to farm, year and geographical region.

\begin{tabular}{|c|c|c|c|c|c|c|}
\hline & \multirow{2}{*}{$\begin{array}{l}\text { Clasificación } \\
\text { A }\end{array}$} & \multicolumn{2}{|c|}{$\mathrm{N}^{\circ}$ Aislados/(\%) total } & \multirow{2}{*}{$\begin{array}{c}\text { Serotipo } 4 \\
1\end{array}$} & \multirow{2}{*}{$\frac{\text { Serotipo } 6}{2}$} & \multirow{2}{*}{$\frac{\text { Serotipo } 7}{6}$} \\
\hline \multirow{9}{*}{ 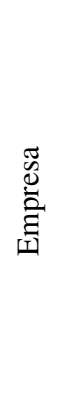 } & & 9 & $(15 \%)^{x y}$ & & & \\
\hline & B & 5 & $(8 \%)^{x y}$ & 1 & 0 & 4 \\
\hline & $\mathrm{C}$ & 5 & $(8 \%)^{x y}$ & 1 & 3 & 1 \\
\hline & $\mathrm{D}$ & 21 & $(35 \%)^{\mathrm{z}}$ & $1^{\mathrm{a}}$ & $3^{\mathrm{a}}$ & $17^{\mathrm{b}}$ \\
\hline & $\mathrm{E}$ & 10 & $(18 \%)^{y}$ & 0 & 6 & 4 \\
\hline & $\mathrm{F}$ & 3 & $(5 \%)^{x y}$ & 1 & 1 & 1 \\
\hline & G & 1 & $(1,7 \%)^{\mathrm{x}}$ & 1 & 0 & 0 \\
\hline & $\mathrm{H}$ & 1 & $(1,7 \%)^{x}$ & 1 & 0 & 0 \\
\hline & I & 5 & $(8 \%)^{x y}$ & 0 & 2 & 3 \\
\hline \multirow{3}{*}{ 究 } & 2007 & 15 & $(25 \%)$ & 2 & 5 & 8 \\
\hline & 2008 & 24 & $(40 \%)$ & 3 & 9 & 12 \\
\hline & 2009 & 21 & $(35 \%)$ & $2^{\mathrm{a}}$ & $3^{\mathrm{a}}$ & $16^{\mathrm{b}}$ \\
\hline \multirow{5}{*}{ 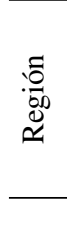 } & Metropolitana & 7 & $(12 \%)^{\mathrm{x}}$ & 2 & 2 & 3 \\
\hline & O’Higgins & 14 & $(23 \%)^{x}$ & $2^{\mathrm{a}}$ & $2^{\mathrm{a}}$ & $10^{\mathrm{b}}$ \\
\hline & Maule & 8 & $(13 \%)^{x}$ & 2 & 4 & 2 \\
\hline & Biobío & 31 & $(52 \%)^{y}$ & $1^{\mathrm{a}}$ & $9^{\mathrm{a}}$ & $21^{\mathrm{b}}$ \\
\hline & Total & 60 & $(100 \%)$ & $7^{\mathrm{a}}$ & $17^{\mathrm{a}}$ & $36^{\mathrm{b}}$ \\
\hline
\end{tabular}

Letras distintas en superíndice (a,b) indican diferencias significativas al evaluar resultados de la misma fila $(\mathrm{P}<0,05)$; $(\mathrm{x}, \mathrm{y}, \mathrm{z})$ indican diferencias estadísticas en resultados de empresa y en resultados por región $(\mathrm{P}<0,05)$.

Different letters in superscript $(\mathrm{a}, \mathrm{b})$ indicate significant differences in results of the same row $(\mathrm{P}<0.05)$; $(\mathrm{x}, \mathrm{y}, \mathrm{z})$ indicate statistical differences between years, and regions $(\mathrm{P}<0.05)$.

o 6, lo que demuestra la presencia de múltiples serotipos de App de estos planteles. Finalmente, en las empresas $\mathrm{G}$ y $\mathrm{H}$ se evaluó tan solo un aislado de App, y en ambas correspondió al serotipo 4. Por otra parte, estadísticamente, la empresa con mayor participación en el estudio fue la empresa D con 21 aislados perteneciente a la Región del Biobío $(\mathrm{P}<0,05)$, mientras que las empresas $\mathrm{G}$ y $\mathrm{H}$ de la Región Metropolitana sólo aportaron un aislado cada una.

Al evaluar la frecuencia de participación de los serotipos según empresa en general no se observaron diferencias estadísticamente significativas, es decir en los planteles no hubo un serotipo predominantemente encontrado. La excepción fue la empresa D en la cual fue aislado con mayor frecuencia el genotipo correspondiente al serotipo $7(\mathrm{P}<0,05)$ (cuadro 3).

Finalmente, el cuadro 4 muestra la presentación de los serotipos de App en todos los planteles evaluados separados por años de muestreo. En ninguna empresa fue posible determinar diferencias estadísticas en la presentación de serotipos de App entre los diferentes años de muestreo.

\section{DISCUSIÓN}

En este estudio se informa sobre la identificación de serotipos de App de unidades de producción intensiva de cerdos. Cada uno de los aislados de App incluidos en el estudio fueron obtenidos desde cerdos con lesiones sospechosas de pleuroneumonía contagiosa porcina, que sugiere que todos los serotipos aislados, el 4, 6 y 7 son capaces de causar el cuadro. De la misma forma, también se confirmó que las pruebas bioquímicas de API $20 \mathrm{NE}$, tienen una correlación de un $100 \%$ con los resultados de PCR, lo que demuestra que la identificación bioquímica fue específica en este estudio.

La serotipificación de los aislados basada en PCR fue eficiente y resolutiva, confirmando lo expuesto por Rayamajhi y col (2005), quienes señalan que esta técnica es reproducible, confiable y rápida de implementar. Asimismo, Oliveira (2008), después de evaluar 75 aislados de campo de App confirmados mediante las técnicas de serotipificación basada en PCR de Rayamajhi y col (2005) y la de hemoaglutinación indirecta (IHA, por sus siglas en inglés), demostró que la técnica de PCR fue más resolutiva que la prueba serológica discriminando todos los serotipos con excepción de los grupos 2/8/15, 9/11 y $12 / 13$, tal como lo señala el autor de la técnica. Oliveira (2008) también evidenció que la prueba de IHA presenta reacciones cruzadas entre los serotipos 1,9 y $11 ; 1$ y $7 ; 7$ y 15 y $3 ; 6$ y 8 , lo cual también ha sido descrito por otros autores como Kucerova y col (2005), sugiriendo que la mejor opción en el desarrollo de la serotipificación de aislados de campo de App podría ser realizada primero 
Cuadro 4. Resultados detallados de serotipificación de cepas de App de acuerdo al año de aislamiento, empresa y ubicación geográfica. Detailed results of serotyping of App strains according to year of diagnosis, farm and location.

\begin{tabular}{cccccc}
\hline Empresa & Año & $\mathrm{N}^{\mathbf{o}}$ Aislados & Serotipo 4 & Serotipo 6 & Serotipo 7 \\
\hline A & 2007 & 3 & 1 & 2 & 0 \\
(R.O.) & 2008 & 4 & 0 & 0 & 4 \\
\hline B & 2009 & 2 & 0 & 0 & 2 \\
\hline (R.O.) & 2007 & 3 & 1 & 0 & 2 \\
C & 2009 & 2 & 0 & 0 & 2 \\
(R.Ma.) & 2007 & 1 & 0 & 0 & 1 \\
\hline & 2008 & 2 & 1 & 1 & 0 \\
D & 2009 & 2 & 0 & 2 & 0 \\
(R B.) & 2007 & 7 & 0 & 2 & 5 \\
\hline & 2008 & 3 & 0 & 0 & 3 \\
E & 2009 & 11 & 1 & 1 & 9 \\
(R.B.) & 2007 & 1 & 0 & 5 & 0 \\
\hline F (R.Ma.) & 2008 & 6 & 0 & 0 & 3 \\
G (R.M.) & 2009 & 3 & 1 & 1 & 1 \\
H (R.M.) & 2008 & 3 & 1 & 0 & 0 \\
I (R.M.) & 2008 & 1 & 1 & 0 & 0 \\
\hline
\end{tabular}

R.M.: Región Metropolitana; R.O.: Región de O’Higgins; R. Ma.: Región del Maule; R.B.: Región del Biobío.

con la técnica basada en PCR y en aquellos aislados que sean clasificados como serotipos 2/8/15 ó 9/11 ó 12/13, se confirme mediante IHA. Es importante destacar que este último punto no pudo ser corroborado en el presente estudio puesto que sólo se utilizó la técnica de PCR para la tipificación.

Por otra parte, se conoce que la distribución de serotipos de App varían dependiendo del país y continente. En América del Sur no existen datos generales publicados, sin embargo, sí existe información por país. En Argentina se han reportado los serotipos $1,3,5,7,8,12$ y 15 (Zielinski 2006), en Brasil los serotipos 3, 5 y 7 son los frecuentemente aislados, aunque también se han descrito los serotipos 1, 4, 6, 9 y 12 (Piffer y col 1997, Kuchiishi y col 2007). Por su parte, situación similar se describe en Perú en donde los serotipos de App descritos hasta la fecha son los serotipos 1, 3, 5 y 7 (Castillo 1996, Mori y col 2010), en Venezuela los serotipos del 1, 2, 3, 4, 5, 6 y 7 , siendo los más prevalentes los serotipos 1, 5 y 7 (Utrera y col 1988, Pineda y col 1996) y en Colombia sólo han sido descritos los serotipos 1, 5 y 7 (Rodríguez-Méndez 2010), en el resto de países sudamericanos exceptuando a Chile no existe información publicada. En resumen en el continente, en general, se han descrito todos los serotipos de App exceptuando los serotipos 10,11, 13 y 14, siendo los más frecuentes los serotipos $1,3,5$ y 7.
En el presente estudio, si bien no se encontraron los aislados correspondientes a los serotipos 1 y 5 , estos ya habían sido anteriormente descritos por Olivares y Morgado (1988), por lo que la situación de Chile sería concordante con lo encontrado en el resto del continente. No obstante, es importante destacar que los serotipos 4, 6 y 7 encontrados en el presente estudio han sido encontrados en otros países de América del Sur, aunque significación clínicopatológica. Marsteller y Fenwick en 1999 y Gottschalk y Taylor en 2006, describen a los serotipos 4, 6 y 7 de App que presentan en general patogenicidad y virulencia leve en comparación a los serotipos 1 y 5 que son más virulentos y probablemente causan mayores problemas en los sistemas de producción intensiva de cerdos en el resto del continente, por lo que probablemente son pesquisados con mayor frecuencia asociados en cuadros clínicos de PCP.

Los resultados indican que la distribución de App en el territorio nacional es conservada, existiendo sólo los serotipos 4, 6 y 7 en las regiones de estudio. Las distribuciones entre regiones y espacio temporal de los aislados en general son homogéneas, evidenciándose al serotipo 7 como el más prevalente. Este resultado fue ayudado por el alto número de aislados clasificados como serotipo $7 \mathrm{del}$ plantel D en la Región del Biobío, único plantel en donde con total legitimidad existió un serotipo predominante, 
debido a la gran cantidad de aislados evaluados. Algo similar aconteció en los planteles de la Región de O'Higgins, A y B, que si bien por sí solos no mostraron un patrón de presentación estadísticamente significativo, sí lo fue al evaluar el resultado por región en donde el serotipo 7 fue el más frecuente. Según Frey (1995), el serotipo 7 posee una virulencia de carácter leve, basado en el perfil de secreción de toxinas que produce, sin embargo, en condiciones de campo, este serotipo ha sido catalogado como un serotipo de difícil control y de alta problemática en las explotaciones porcinas, siendo su patogenicidad muy alta (Tadjine y Mittal 2001). Esta diferencia de patogenicidad entre un mismo serotipo es concordante con lo descrito por Gottschalk y Taylor (2006), quienes mencionan que existen serotipos que pueden ser altamente virulentos en una región geográfica, mientras que en otras no representan una gran problemática. Como se mencionó anteriormente, si bien los aislados del presente estudio no fueron diagnosticados desde brotes importantes de PCP, sí fueron recopilaron desde lesiones pleuroneumonías, por lo que estas eventualmente pueden ser un problema en las explotaciones porcinas nacionales.

Anteriormente, Olivares y Morgado (1988) reportaron los serotipos 1 y 5 en la Región Metropolitana, no encontrados en este estudio, hecho no implica que no se encuentren hoy en el país. La no detección de estos serotipos probablemente se deba a que la cantidad de muestras recolectadas en la Región Metropolitana no representa la situación real de la región, aunque confirma que estos no se diagnostican frecuentemente en la actualidad y sugiere que pudieron haber sido eliminados de las explotaciones chilenas, ya que si estuvieran presentes probablemente provocarían cuadros clínicos de relevancia, tal como ocurre en el resto de los países vecinos.

El hecho de que en la mayoría de los planteles se haya detectado más de un serotipo de App no es una situación nueva, ya que ha sido descrito con anterioridad, por Dubreuil y col (2000) y Gottschalk (2003) quienes reportaron que granjas porcinas pueden tener más de un serotipo en su población y que es una situación común en los planteles de producción intensiva. Esto concuerda con los resultados obtenidos en este estudio, especialmente en las empresas A, C, D y F en las que se detectaron los tres serotipos, las cuales se ubican en las regiones de O'Higgins, Maule y Biobío. En otras tres empresas porcinas (B, E e I), ubicadas en las regiones Metropolitana, de O'Higgins y Biobío, se identificaron dos serotipos en cada una de ellas, mientras que para las empresas $\mathrm{G}$ y $\mathrm{H}$ sólo se detectó un solo serotipo. En estas dos últimas empresas, el número de aislados presentes fue solo uno, por lo que el serotipo identificado en cada una de ellas puede que no sea el único y no representa el estatus real del sistema productivo.

Finalmente, los resultados en general sugieren que probablemente los serotipos 4, 6 y 7 están presentes hace bastante tiempo en los planteles y se han mantenido sin grandes variaciones y probablemente sin la introducción de nuevos serotipos, sin embargo, no fue posible conocer la variabilidad de serotipos por años dentro de un plantel, debido a la escasa cantidad de aislados.

Este es el primer reporte de App en Chile utilizando la serotipificación en base a perfiles genéticos mediante técnicas moleculares, demostrando la variedad de serotipos que se encuentran en las principales zonas de producción porcina de nuestro país. Futuros estudios son necesarios con el fin de conocer de mejor forma la dinámica de presentación de serotipos en los planteles, en los que se debe considerar un mayor número de aislados a evaluar.

\section{RESUMEN}

Actinobacillus pleuropneumoniae (App) es el agente etiológico de la pleuroneumonía contagiosa porcina, una de las enfermedades de etiología bacteriana de mayor relevancia en producción porcina. En el mundo se han descrito 15 serotipos de App, en Chile solo los serotipos 1 y 5 . La serotipificación requiere mucho tiempo, trabajo y dinero, actualmente se encuentran herramientas moleculares para realizar una "serotipificación" mediante la genotipificación de toxinas Apx. Así, se evaluaron 60 aislados de App provenientes de nueve empresas porcinas de producción intensiva distribuidas en distintas regiones de Chile, obtenidas desde pulmones de cerdos con lesiones compatibles con pleuroneumonía contagiosa porcina. Las bacterias fueron aisladas mediante los métodos tradicionales y confirmados por API, recolectados durante los años 2007, 2008 y 2009. Los resultados identificaron los genotipos correspondientes sólo a los serotipos 4,6 y 7, los cuales se describen por primera vez en Chile, siendo el más frecuente el serotipo 7. En las diferentes zonas estudiadas, no existió un serotipo predominante, excepto en las regiones de O'Higgins y del Biobío en las cuales fue más frecuentemente aislado el serotipo 7. El presente estudio es el primer acercamiento con el fin de conocer la distribución de serotipos de App en Chile. Con el fin de conocer la real diversidad genética y serotipos de App en los diversos planteles en Chile es necesario realizar estudios que contemplen un mayor número de aislados.

\section{AGRADECIMIENTOS}

Los autores agradecen la colaboración prestada a Aura Villamil, del Laboratorio de Diagnóstico Porcino de la Facultad de Medicina Veterinaria de la Universidad de Concepción y a Macarena Cortez Jara por su apoyo en la revisión y corrección del trabajo.

\section{REFERENCIAS}

Castillo C. 1996. Aislamiento y determinación de serotipos de Actinobacillus pleuropneumoniae en porcinos beneficiados en camal de Lima. Tesis de Médico Veterinario. Facultad de Medicina Veterinaria, Universidad Nacional Pedro Ruiz Gallo, Lambayeque, Perú.

Dubreuil D, M Jacques, KR Mittal, M. Gottschalk. 2000. Actinobacillus pleuropneumoniae surface polysaccharides: their role in diagnosis and immunogenicity. Anim Health Res Rev 1, 73-93.

Frey J. 1995. Virulence in Actinobacillus pleuropneumoniae and RTX toxins. Trends Microbiol 3, 257-261.

Gottschalk M, A Broes, K Mittal, M Kobisch, P Kuhnert, A Lebrun, J Frey. 2003. Non-pathogenic Actinobacillus isolates antigenically and biochemically similar to Actinobacillus pleuropneumoniae: a novel species?. Vet Microbiol 92, 87-101.

Gottschalk M, D Taylor. 2006. Actinobacillus pleuropneumoniae. In: BE Straw BE, Zimmerman JJ, D' Allaire S, Taylor DJ (eds). Diseases of swine. $9^{\text {th }}$ ed. Blackwell Publishing, Iowa, USA, Pp 563-576. 
Haesebrouck F, K Chiers, I Van Overbeke, R Ducatelle. 1997. Actinobacillus pleuropneumoniae infections in pigs: the role of virulence factors in the pathoghenesis and protection. Vet Microbiol 58, 239-249.

Kucerova Z, R Jaglic, R Ondriasova, K Nedbalcova. 2005. Serotype distribution of Actinobacillus pleuropneumoniae in Czech Republic during period 2003-2004. Vet Med Czech 50, 355-360.

Kuchiishi S, J Kich, M Fracasso, D Spricigo, C Klein, M Fávero, I Piffer. 2007. Sorotipos de Actinobacillus pleuropneumoniae isolados no Brasil de 1993 a 2006. Acta Scientiae Veterinariae 35,79-82.

Marsteller T, B Fenwick. 1999. Actinobacillus pleuropneumoniae disease and serology. Swine Health Prod 4, 161-165.

Mori L, S Calle, C Pinto, M Torres, N Falcón, S Morales. 2010. Frecuencia de infección con Actinobacillus pleuropneumoniae en granjas porcinas tecnificadas de la costa peruana. Rev Investig Vet Perú 21,124-129.

Nielsen R, LO Andersen, T Plambeck, JP Nielsen, LT Krarup, SE Jorsal. 1997. Serological characterization of Actinobacillus pleuropneumoniae biotype 2 strains isolated from pigs in two Danish herds. Vet Microbiol 54, 35-41.

Olivares P, A Morgado. 1988. Isolation and serotyping of Haemophilus pleuropneumoniae in three porcine pleuropneumonia outbreaks in central Chile. Arch Med Vet 20, 147-152.

Oliveira S. 2008. Diagnostic update on Actinobacillus pleuropneumoniae. In: Pijoan C (ed). International Symposium: New solutions to old problems. Saint Paul, MN, USA, Pp 15-23.

Piffer IA, CS Klein , M Favero, J Figueiredo. 1997. Caracterização bioquímica e sorológica de 55 amostras de A. pleuropneumoniae isoladas no Brasil. Arq Bras Med Vet Zootec 49, 123-129.

Pineda Y, A de Lopez, F de Aponte, C de Parra, J Santander. 1996. Serotipos de Actinobacillus pleuropneumoniae aislados de cerdos en Venezuela y su susceptibilidad a los agentes antimicrobianos Vet Trop 21, 35-47.
Rayamajhi N, SJ Shin, SG Kang, DY Lee, JM Ahn, HS Yoo. 2005. Development and use of a multiplex polymerase chain reaction assay based on Apx toxin genes for genotyping of Actinobacillus pleuropneumoniae isolates. J Vet Diagn Invest 17, 359-362.

Rodríguez-Méndez G. 2010. Detección e identificación simultánea y discriminación del Actinobacillus pleuropneumoniae serotipos 1, 5 y 7 por técnicas de PCR múltiples basadas en genes apxIVA y cps. Tesis magistral, Facultad de Ciencias, Pontificia Universidad Javierana, Bogotá, Colombia.

Sánchez M, L Cuevas, A Morgado. 1986. Pleuroneumonía porcina: aislamiento de Haemophilus pleuropneumoniae en Chile. Av Cs Vet 1, 45-50.

Savoye C, J Jobert, F Berthelot-Hérault, A Keribin, R Cariolet, H Morvan, F Madec, M Köbisch. 2000. A PCR assay used to study aerosol transmission of Actinobacillus pleuropneumoniae from samples of live pigs under experimental conditions. Vet Microbiol 73, 227-347.

Schaller A, S Djordjevic, G Eamens, W Forbes, R Kuhn, P Kuhnert, M Gottschalk, J Nicolet, J Frey. 2001. Identification and detection of Actinobacillus pleuropneumoniae by PCR based on the gene apxIVA. Vet Microbiol 79, 47-62.

Tadjine M, K Mittal. 2001. Study of antigenic heterogeneity among Actinobacillus pleuropneumoniae serotype 7 strains. Vet Microbiol 78, 49-60.

Thacker EL, R Thanawongnuwech. 2002. Porcine respiratory disease complex (PRDC). Thai J Vet Med 32, 126-134.

Utrera V, A Gallardo, L Mariño. 1988. Serotipificación de Haemophilus (Actinobacillus) pleuropneumoniae. Vet Trop 13, 43-54.

Zielinski GC. 2006. Actinobacillus pleuropneumoniae: situación en Argentina. En: Memorias del V Congreso de Producción Porcina del Mercosur, Río Cuarto, Argentina, Pp 225-231. 